PROCEEDINGS OF THE

AMERICAN MATHEMATICAL SOCIETY

Volume 133, Number 5, Pages 1563-1567

S 0002-9939(04)07694-4

Article electronically published on November 22, 2004

\title{
AN EXTENSION OF BIRAN'S LAGRANGIAN BARRIER THEOREM
}

\author{
GUANG-CUN LU
}

(Communicated by Jon G. Wolfson)

\begin{abstract}
We use the Gromov-Witten invariants and a nonsqueezing theorem by the author to affirm a conjecture by P. Biran on the Lagrangian barriers.
\end{abstract}

\section{MAin RESUlts}

A Kähler manifold is a triple consisting of a symplectic manifold $(M, \omega)$ and an integrable complex structure $J$ compatible with $\omega$ on $M$. If $[\omega] \in H^{2}\left(M^{2 n}, \mathbb{Z}\right)$, it follows from Kodaira's embedding theorem that there exists a smooth and reduced complex hypersurface $\Sigma \subset M$ such that its homology class $[\Sigma] \in H_{2 n-2}(M)$ represents the Poincaré dual $k[\omega] \in H^{2}(M)$ for some $k \in \mathbb{N}$. Following [1] $\mathcal{P}=$ $(M, \omega, J ; \Sigma)$ is called a smoothly polarized Kähler manifold. Under the conditions that either $\operatorname{dim}_{\mathbb{R}} M \leq 6$ or $\left.\omega\right|_{\pi_{2}(M)}=0$ the following two theorems were proved in Theorem 1.D and Theorem 4.A of [1] respectively.

Theorem 1.1. If $(M, \omega)$ is a Kähler manifold with $[\omega] \in H^{2}(M, \mathbb{Q})$, then for every $\epsilon>0$ there exists a Lagrangian $C W$-complex $\triangle_{\epsilon} \subset(M, \omega)$ such that every symplectic embedding $\varphi: B(\epsilon) \rightarrow(M, \omega)$ must satisfy $\varphi(B(\epsilon)) \cap \triangle_{\epsilon} \neq \emptyset$.

Theorem 1.2. If $\mathcal{P}=(M, \omega, J ; \Sigma)$ is an n-dimensional polarized Kähler manifold of degree $k$, then every symplectic embedding $\varphi: B^{2 n}(\lambda):=\left\{x \in \mathbb{R}^{2 n}|| x \mid \leq \lambda^{2}\right\} \rightarrow$ $(M, \omega)$ with $\lambda^{2} \geq \frac{1}{k \pi}$ must intersect the skeleton $\triangle_{\mathcal{P}}$ associated to the polarization $\mathcal{P}$.

For the definitions of $\triangle_{\epsilon}$ and $\triangle_{\mathcal{P}}$ the reader may refer to [1]. Actually, such generalizations were conjectured in Remark 4.B of [1. As in [1] Theorem 1.1 may be derived from Theorem 1.2.

Following [2] a Stein manifold is said to be subcritical if it admits a plurisubharmonic function that has only critical points of index less than half the real dimension. A polarization $\mathcal{P}=(M, \omega, J ; \Sigma)$ is called subcritical if the complement $(M \backslash \Sigma, \omega)$ is a subcritical Stein manifold. From the proof of Theorem 1.2 we easily get the following generalizations of Theorem $\mathrm{F}$ and Theorem $\mathrm{G}$ in [2].

Received by the editors July 28, 2003 and, in revised form, January 15, 2004.

2000 Mathematics Subject Classification. Primary 57R17, 53D35, 53D40; Secondary 32Q15, $32 \mathrm{Q} 28$.

Key words and phrases. Polarized Kähler manifolds, Lagrangian barrier, Gromov-Witten invariants, nonsqueezing theorem, Gromov width.

The author was supported in part by NNSF 19971045 and 10371007 of China.

(C)2004 American Mathematical Society Reverts to public domain 28 years from publication 
Theorem 1.3. If a closed Kähler manifold $(M, \omega, J)$ admits a subcritical polarization $\mathcal{P}=(M, \omega, J ; \Sigma)$ of degree $k$, then $\mathcal{W}_{G}(M, \omega) \leq \frac{1}{k}$ and $k \leq \operatorname{dim}_{\mathbb{C}} M$. Moreover, if the linear system of holomorphic sections of the normal bundle to $\Sigma, N_{\Sigma / M} \rightarrow \Sigma$, is base point free, then $\mathcal{W}_{G}(M, \omega)=\frac{1}{k} \geq 1 / \operatorname{dim}_{\mathbb{C}} M$. Here $\mathcal{W}_{G}(M, \omega)$ stands for the Gromov width of $(M, \omega)$.

\section{Proof of the theorems}

Our purpose is to prove Theorem 1.2. The ideas are similar to those of Biran. However, we use the theory of virtual cycles and some techniques in [4] to compute the desired the Gromov-Witten invariant without additional assumptions as in [1]. Then we directly use the author's previous work on pseudo-symplectic capacities and avoid Gromov's arguments as used in [1]. For convenience of the reader we need to recall some related notions in [1. A subset $\triangle$ of a symplectic manifold $(M, \omega)$ is called an embedded $C W$-complex if there exists an abstract finite and path-connected CW-complex $K$ and a homeomorphism $i: K \rightarrow \triangle \subset M$ such that for every cell $C \subset K$ the restriction $\left.i\right|_{\operatorname{Int}(C)}: \operatorname{Int}(C) \rightarrow M$ is a smooth embedding. When the image $i(\operatorname{Int}(C))$ by $i$ of each cell $C$ in $K$ is an isotropic submanifold of $(M, \omega)$ the above embedded $\mathrm{CW}$-complex $\triangle$ is called isotropic. The dimension $\operatorname{dim} \triangle$ of $\triangle$ is defined as the maximum of those of cells in $K$. An embedded isotropic $\mathrm{CW}$-complex of dimension $\frac{1}{2} \operatorname{dim}_{\mathbb{R}} M$ in $(M, \omega)$ is called a Lagrangian $\mathrm{CW}$-complex.

Another key role in [1] is the standard symplectic disc bundle. For a closed symplectic manifold $(S, \sigma)$ with $[\sigma] \in H^{2}(S ; \mathbb{Z})$ there exists a Hermitian line bundle $p: L \rightarrow S$ with $c_{1}(L)=[\sigma]$ and a compatible connection $\nabla$ on $L$ with curvature $R^{\nabla}=2 \pi i \sigma$ (cf. [5 Prop. 8.3.1]). Denote by $\|\cdot\|$ the Hermitian metric and by $E_{L}=\{v \in L \mid\|v\|<1\}$ the open unit disc bundle of $L$. Let $\alpha^{\nabla}$ be the associated transgression 1-form on $L \backslash 0$ with $d \alpha^{\nabla}=-p^{*} \sigma$, and $r$ the radial coordinate along the fibres induced by $\|\cdot\|$. It was shown that

$$
\omega_{\text {can }}:=p^{*} \sigma+d\left(r^{2} \alpha^{\nabla}\right)
$$

is a symplectic form on $E_{L}$ and that the symplectic type of $\left(E_{L}, \omega_{\text {can }}\right)$ depends only on the symplectic type of $(S, \sigma)$ and the topological type of the complex line bundle $p: L \rightarrow S$. Moreover, $\left(E_{L}, \omega_{\text {can }}\right)$ is uniquely characterized (up to symplectomorphism) by the following three properties:

- All fibres of $p: E_{L} \rightarrow S$ are symplectic with respect to $\omega_{\text {can }}$ and have area 1.

- The restriction of $\omega_{\text {can }}$ to the zero section $S \subset E_{L}$ equals $\sigma$.

- $\omega_{\text {can }}$ is $S^{1}$-invariant with respect to the obvious circle action on $E_{L}$.

Following [1] we call

$$
p:\left(E_{L}, \omega_{\text {can }}\right) \rightarrow(S, \sigma)
$$

a standard symplectic disc bundle over $(S, \sigma)$ modelled on $L$ and $p:\left(E_{L}, c \omega_{\text {can }}\right) \rightarrow$ $(S, \sigma)$ a standard symplectic disc bundle with fibres of area $c$ for each $c>0$.

As was done in [1] this standard symplectic disc bundle can be compactified into a $\mathbb{C} P^{1}$-bundle over $(S, \sigma)$. Indeed, let $\mathbb{C}$ stand for the trivial complex line bundle over $S$. Then the direct sum $L \oplus \mathbb{C}$ is a complex vector bundle of rank 2 over $S$. Denote by $p_{X}: X_{L}=P(L \oplus \mathbb{C}) \rightarrow S$ its projective bundle, which is a $\mathbb{C} P^{1}$-bundle over $S$. It has two distinguished sections: the zero section $Z_{0}=P(0 \oplus \mathbb{C})$ and the section at infinity $Z_{\infty}=P(L \oplus 0)$. Clearly, the open manifold $X_{L} \backslash Z_{\infty}$ is diffeomorphic to the disc bundle $E_{L}$. Let $F_{s}$ stand for the fibre of $X_{L}$ at $s \in S$. It 
was shown in [1] that a given $J_{S} \in \mathcal{J}(S, \sigma)$ and the connection $\nabla$ determine a unique almost complex structure $J_{L}$ on the total space of $L$. This $J_{L}$ induces the almost complex structures $J_{E}$ on $E$ and $J_{X}$ on $X_{L}$ again. In particular, $J_{E} \in \mathcal{J}\left(E_{L}, \omega_{\text {can }}\right)$ and $Z_{0}, Z_{\infty}$ and all fibres $F_{s}$ of $p_{X}: X_{L} \rightarrow S$ are holomorphic with respect to $J_{X}$. These show that $p_{X}:\left(X_{L}, J_{X}\right) \rightarrow\left(S, J_{S}\right)$ is an almost complex fibration with fibre $\left(\mathbb{C} P^{1}, i\right)$ in the following sense.

Definition 2.1 ( 1 , Def. 6.3.A]). Let $\left(F, J_{F}\right),\left(B, J_{B}\right)$ and $(X, J)$ be three almost complex manifolds. A holomorphic map $p:(X, J) \rightarrow\left(B, J_{B}\right)$ is called an almost complex fibration with fibre $\left(F, J_{F}\right)$ if every $b \in B$ has a neighborhood $U$ and a trivialization $\varphi:\left.X\right|_{U} \rightarrow U \times F$ such that for every $a \in U$ the map $\left.\varphi\right|_{F_{a}}$ : $\left(F_{a},\left.J\right|_{F_{a}}\right) \rightarrow\left(a \times F, J_{F}\right)$ is holomorphic.

For every $0<\rho<1$ both $E_{L}(\rho):=\left\{v \in E_{L} \mid\|v\|<\rho\right\}$ and $\overline{E_{L}(\rho)}:=\{v \in$ $\left.E_{L} \mid\|v\| \leq \rho\right\}$ are subbundles of $E_{L}$.

Lemma $2.2\left(\left[1\right.\right.$, Lem. 5.2.A]). Let $p:\left(E_{L}, \omega_{\text {can }}\right) \rightarrow(S, \sigma)$ be a standard symplectic disc bundle modeled on a Hermitian line bundle $p: L \rightarrow S$ as above. Then there exists a diffeomorphism $f: E_{L} \rightarrow X_{L} \backslash Z_{\infty}$ and a family of symplectic forms $\left\{\eta_{\rho}\right\}_{0<\rho<1}$ on $X_{L}$ such that:

(1) $f^{*} \eta_{\rho}=\omega_{\text {can }}$ on $\overline{E_{L}(\rho)}$ for every $0<\rho<1$.

(2) $f$ sends the fibres of $E_{L} \rightarrow S$ to the fibres of $X_{L} \backslash Z_{\infty} \rightarrow S$.

(3) If $S$ is identified with the zero-section of $E_{L}$, then $f(S)=Z_{0}$ and $\left.p_{X} \circ f\right|_{S}$ : $S \rightarrow S$ is the identity map.

(4) $Z_{0}, Z_{\infty}$ and all fibres $F_{s}=p_{X}^{-1}(s)(s \in S)$ of $p_{X}: X_{L} \rightarrow S$ are not only symplectic with respect to $\eta_{\rho}$ for every $0<\rho<1$, but also holomorphic with respect to $J_{X}$. Moreover, for every $0<\rho<1$ it follows that $\left.\eta_{\rho}\right|_{T Z_{0}}=$ $\left.\left(p_{X}^{*} \sigma\right)\right|_{T Z_{0}}$ and $\left.\eta_{\rho}\right|_{T Z_{\infty}}=\left.c_{\rho}\left(p_{X}^{*} \sigma\right)\right|_{T Z_{\infty}}$ for some $0<c_{\rho}<1-\rho^{2}$.

(5) The area of the fibres $F_{s}$ satisfies $\rho^{2}<\int_{F_{s}} \eta_{\rho}<1$ for every $0<\rho<1$.

Moreover, if $J_{S} \in \mathcal{J}(S, \sigma)$, then $J_{X} \in \mathcal{J}\left(X_{L}, \eta_{\rho}\right)$ for every $0<\rho<1$. In particular, if $\left(S, \sigma, J_{S}\right)$ is Kähler and $L \rightarrow S$ is a holomorphic line bundle, then $\left(X_{L}, \eta_{\rho}, J_{X}\right)$ is Kähler for every $0<\rho<1$.

It was proved in Lemma 6 . A of 1 that the almost complex structure $J_{X}$ is regular for the class of the fibre $F \in H_{2}\left(X_{L}, \mathbb{Z}\right)$ and that the space of $J_{X}$-holomorphic spheres in the class $F$ is made up of exactly the fibres $F_{s}=p_{X}^{-1}(s), s \in S$. So the space $\bar{M}_{0,3}\left(X_{L}, J_{X}, F\right)$ of all $J_{X}$-holomorphic stable maps in class $F$ of genus 0 and with 3 marked points is nonempty. As expected we have:

Lemma 2.3. Let $F \in H_{2}\left(X_{L} ; \mathbb{Z}\right)$ denote the homology class of a fibre of $X_{L} \rightarrow S$. Then the Gromov-Witten invariant of $\left(X_{L}, \eta_{\rho}\right)$,

$$
\Psi_{F, 0,3}^{\left(X_{L}, \eta_{\rho}\right)}\left(p t ;\left[Z_{0}\right],\left[Z_{\infty}\right], p t\right)=1 .
$$

That is, $\left(X_{L}, \eta_{\rho}\right)$ is a strong 0-symplectic uniruled manifold in the sense of Definition 1.13 in 3 .

Proof. Let $A=\left[\mathbb{C} P^{1}\right] \in H_{2}\left(\mathbb{C} P^{1} ; \mathbb{Z}\right)$ and $i: \mathbb{C} P^{1} \rightarrow X_{L}$ be the inclusion. Then $F=$ $i_{*}(A)$. Since the Gromov-Witten invariants are symplectic deformation invariants, we may fix an $\eta \equiv \eta_{\rho}$. By Lemma 2.2 all fibres $F_{s}=\pi_{X}^{-1}(s)(s \in S)$ are not only symplectic with respect to $\eta$ but also holomorphic with respect to $J_{X}$. Thus for each $s \in S$ and $x \in F_{s}$ the tangent space $T_{x} F_{s}$ is a symplectic and $J_{X}(x)$-invariant 
subspace of $\left(T_{x} X_{L}, \eta_{x}\right)$. Let $H_{x}$ denote the $\eta_{x}$-orthogonal complement of $T_{x} F_{s}$. Then $T_{x} X_{L}=H_{x} \oplus T_{x} F_{s}$ and $\left.D p_{X}(x)\right|_{H_{x}}: H_{x} \rightarrow T_{s} S$ is an isomorphism. Note that $J_{X}$ is also compatible with $\eta$. We have that $\eta\left(J_{X} \xi, J_{X} \eta\right)=\eta(\xi, \eta)$ for all $\xi, \eta \in T X_{L}$. This implies that $H_{x}$ is also a $J_{X}(x)$-invariant subspace of $T_{x} X_{L}$. Consequently, $J_{X}(x)$ preserves the splitting $T_{x} X_{L}=H_{x} \oplus T_{x} F_{s}$. Moreover, the projection $p_{X}:\left(X_{L}, J_{X}\right) \rightarrow\left(S, J_{S}\right)$ is holomorphic (cf. [1, $\left.\left.\S 6.3\right]\right)$. Hence the almost complex structure $J_{X}$ on $X_{L}$ is fibered in the sense of Definition 2.2 in [4].

Since any stable map has connected image set it is easily checked that each stable map in $\bar{M}_{0,3}\left(X_{L}, J_{X}, F\right)$ must also entirely lie in a fibre of $X_{L}$. It follows from this and Lemma 4.3 in [4] that for any representative $\tilde{\tau}=(\Sigma, \tilde{h})$ of $\tau \in \bar{M}_{0,3}\left(X_{L}, J_{X}, F\right)$ the cokernel of $D \bar{\partial}_{J_{X}}(\tilde{h})$ can be spanned by elements of the space

$$
\mathcal{L}_{\tilde{h}}^{V}=L^{1, p}\left(\wedge^{0,1}\left(\tilde{h}^{*}\left(T_{\text {vert }} X_{L}\right)\right)\right),
$$

where $V=T_{\text {vert }} X_{L}$ is the vertical tangent bundle of $T X_{L}$. By Proposition 4.3 in 4 one can choose $R$ and the embeddings $e$ in the construction of the virtual moduli cycle $\bar{M}_{0,3}^{\nu}\left(X_{L}, J_{X}, F\right)$ of $\bar{M}_{0,3}\left(X_{L}, J_{X}, F\right)$ such that $e_{\tilde{\tau}}(\nu) \in \mathcal{L}_{\tilde{h}}^{V}$ for all $\nu \in R$ and all $\tilde{\tau}$. That is, we can choose a fibered pair $\left(J_{X}, \nu\right)$ on $X_{L}$ in the sense of Definition 4.4 in [4]. As proved in Proposition 4.4 of [4] such a virtual moduli cycle $\bar{M}_{0,3}^{\nu}\left(X_{L}, J_{X}, F\right)$ has the following property. For each element of it, a parameterized stable map $(\Sigma, \tilde{h})$, each component $\tilde{h}_{k}$ of $\tilde{h}$ satisfies an equation of the form $\bar{\partial}_{J_{X}} \tilde{h}_{k}=\nu_{k}$ for some section $\nu_{k}$ of $\wedge^{0,1}\left(\tilde{h}_{k}^{*}\left(T_{\text {vert }} X_{L}\right)\right)$. This implies that each $p_{X} \circ \tilde{h}_{k}: S^{2} \rightarrow S$ is $\left(i, J_{S}\right)$-holomorphic, and therefore that each element in $\bar{M}_{0,3}^{\nu}\left(X_{L}, J_{X}, F\right)$ has the image contained in a single fibre of $X_{L}$. For any $s \in$ $S$ we identify $\left(F_{s},\left.J_{X}\right|_{F_{s}}\right)$ with $\left(\mathbb{C} P^{1}, i\right)$. Then the subset $\bar{M}_{0,3}^{\nu}\left(X_{L}, J_{X}, F\right)_{s}$ of $\bar{M}_{0,3}^{\nu}\left(X_{L}, J_{X}, F\right)$ consisting of stable maps with image in $F_{s}$ regularizes the space $\bar{M}_{0,3}\left(\mathbb{C} P^{1}, i, A\right)$ in the sense of Definition 4.3 in 4 . Hence

$$
\Psi_{F, 0,3}^{\left(X_{L}, \eta\right)}\left(p t ;\left[Z_{0}\right],\left[Z_{\infty}\right], p t\right)=\Psi_{A, 0,3}^{\left(\mathbb{C} P^{1}, \omega_{F S}\right)}(p t ; p t, p t, p t)=1 .
$$

Here we have used $p t$ to stand for the point classes in the different spaces. Lemma 2.3 is proved.

Recall that for a closed symplectic manifold $(M, \omega)$ and homology classes $\alpha_{0}, \alpha_{\infty}$ $\in H_{*}(M, \mathbb{Q})$ we, in [3. Def. 1.8], defined a number

$$
\mathrm{GW}_{g}\left(M, \omega ; \alpha_{0}, \alpha_{\infty}\right) \in(0,+\infty]
$$

by the infimum of the $\omega$-areas $\omega(A)$ of the homology classes $A \in H_{2}(M ; \mathbb{Z})$ for which the Gromov-Witten invariant $\Psi_{A, g, m+2}\left(C ; \alpha_{0}, \alpha_{\infty}, \beta_{1}, \cdots, \beta_{m}\right) \neq 0$ for some homology classes $\beta_{1}, \cdots, \beta_{m} \in H_{*}(M ; \mathbb{Q})$ and $C \in H_{*}\left(\overline{\mathcal{M}}_{g, m+2} ; \mathbb{Q}\right)$ and integer $m>$ 0 . Moreover, in Definition 1.25 of [3] we also defined another number $\operatorname{GW}(M, \omega) \in$ $(0,+\infty]$ by

$$
\operatorname{GW}(M, \omega)=\inf \operatorname{GW}_{g}(M, \omega ; p t, \alpha),
$$

where the infimum is taken over all nonnegative integers $g$ and all homology classes $\alpha \in H_{*}(M ; \mathbb{Q}) \backslash\{0\}$ of degree $\operatorname{deg} \alpha \leq \operatorname{dim} M-1$. Theorem 1.26 in [3] claimed that

$$
\mathcal{W}_{G}(M, \omega) \leq \mathrm{GW}(M, \omega)
$$

for any symplectic uniruled manifold $(M, \omega)$ of dimension at least 4 . 
Proof of Theorem 1.2. By the decomposition Theorem 2.6.A in [1], $\left(M \backslash \triangle_{\mathcal{P}}, \omega\right)$ is symplectomorphic to the standard symplectic disc bundle $\left(E_{N_{\Sigma}}, \frac{1}{k} \omega_{\text {can }}\right) \rightarrow(\Sigma, \sigma=$ $\left.k \omega\right|_{\Sigma}$ ) over $\Sigma$, which is modeled on the normal bundle $N_{\Sigma}$ and with fibres of area $1 / k$. Assume that $\varphi(B(\lambda)) \cap \triangle_{\mathcal{P}}=\emptyset$ for some symplectic embedding $\varphi: B^{2 n}(\lambda) \rightarrow$ $(M, \omega)$ with $\lambda^{2} \geq \frac{1}{k \pi}$. Then there exists a symplectic embedding $\psi: B(\lambda) \rightarrow$ $\left(E_{N_{\Sigma}}, \frac{1}{k} \omega_{\text {can }}\right)$. Since $\psi(B(\lambda))$ is compact there exists a positive number $\rho \in(0,1)$ such that $\psi(B(\lambda)) \subset E_{N_{\Sigma}}(\rho)$. Lemma 2.2 gives a symplectic embedding from $\psi(B(\lambda))$ into $\left(X_{N_{\Sigma}}, \frac{1}{k} \eta_{\rho}\right)$. Hence

$$
\mathcal{W}_{G}\left(X_{N_{\Sigma}}, \frac{1}{k} \eta_{\rho}\right) \geq \pi \lambda^{2} \geq 1 / k
$$

On the other hand, by the definition of $\operatorname{GW}(M, \omega)$ and Lemmas 2.2, 2.3 we have

$$
\operatorname{GW}\left(X_{N_{\Sigma}}, \eta_{\rho}\right) \leq \int_{F_{s}} \eta_{\rho}<1
$$

for this $\rho$. This and (1) together give

$$
\mathcal{W}_{G}\left(X_{N_{\Sigma}}, \frac{1}{k} \eta_{\rho}\right) \leq \mathrm{GW}\left(X_{N_{\Sigma}}, \frac{1}{k} \eta_{\rho}\right) \leq \frac{1}{k} \int_{F_{s}} \eta_{\rho}<\frac{1}{k},
$$

which contradicts (2). Theorem 1.2 is proved.

\section{ACKNOWLEDGEMENTS}

I am grateful to Kai Cieliebak for sending me some preprints. I would like to thank the referees for much advice for improving the presentation, and for pointing

out a careless mistake in the statement of Theorem 1.3 in the previous version of the paper.

\section{REFERENCES}

[1] P. Biran, Lagrangian barriers and symplectic embeddings, Geom. Funct. Anal. 11 (2001), 407-464. MF 1844078 (2002g:53153)

[2] P. Biran and K. Cieliebak, Symplectic topology on subcritical manifolds, Comment. Math. Helvetici 76 (2001), 712-753. MR1881704 (2003b:53091)

[3] G. C. Lu, Gromov-Witten invariants and pseudo symplectic capacities, math.SG/0103195, v7, May 21, 2004.

[4] D. McDuff, Quantum homology of fibrations over $S^{2}$, International Journal of Mathematics, 11 (2000), 665-721. MR1780735 (2001i:53157)

[5] N. M. J. Woodhouse, Geometric Quantization, Second edition, Oxford University Press, New York, 1991. MR 1183739|(94a:58082)

Department of Mathematics, Beijing Normal University, Beijing 100875, People's Republic of China

E-mail address: gclu@bnu.edu.cn 